\title{
Clinical characteristics and disease outcome of patients with non-medullary thyroid cancer and brain metastases
}

\author{
ILANA SLUTZKY-SHRAGA ${ }^{1,2}$, ALEX GORSHTEIN ${ }^{1,2}$, AHARON POPOVITZER ${ }^{2,3}$, EYAL ROBENSHTOK ${ }^{1,3}$, \\ GLORIA TSVETOV $^{1,2}$, AMIT AKIROV ${ }^{1,2}$, DANIA HIRSCH $^{1,2}$ and CARLOS BENBASSAT ${ }^{2,4}$ \\ ${ }^{1}$ Endocrine Institute, Rabin Medical Center, Beilinson Hospital, Petach Tikva 4941492; ${ }^{2}$ Sackler Faculty of Medicine, \\ Tel Aviv University, Tel Aviv 6997801; ${ }^{3}$ ENT Oncology Clinic, Rabin Medical Center, Beilinson Hospital, \\ Petach Tikva 4941492; ${ }^{4}$ Endocrine Institute, Assaf HaRofeh Medical Center, Zerifin 70300, Israel
}

Received September 10, 2016; Accepted September 22, 2017

DOI: $10.3892 / \mathrm{ol} .2017 .7325$

\begin{abstract}
Brain metastases from non-medullary thyroid carcinoma (NMTC) are rare, with a reported frequency of $\sim 1 \%$, and patient survival time is $<1$ year after diagnosis. The optimal management of brain metastases in this setting continues to be debated. The aim of the present study was to evaluate a series of patients with brain metastases from NMTC attending a single tertiary medical center. The electronic database of Rabin Medical Center was reviewed for all patients with NMTC and distant metastases who were diagnosed and treated between 1970 and 2014. Those with brain metastases were identified and formed the study group. Data were collected from medical records comprising clinicopathological characteristics, time intervals for diagnosis and treatment, treatment modalities and outcome. Of the 172 patients with NMTC and distant metastases, 10 possessed brain metastases. These included 6 females and 4 males of median age 53.5 years (range, 18-81 years). All patients had lung metastases and 7 demonstrated bone metastases. The median interval between the diagnoses of NMTC and brain metastases was 40 months (range, 9-207 months). Of the 10 patients, 1 presented with brain metastases at primary diagnosis. Treatment of the brain metastases consisted of surgery, radiotherapy (external beam, stereotactic), and radioiodine, alone or in combination. A total of 2 patients received tyrosine kinase inhibitors. The median overall survival time from diagnosis of brain metastasis was 15 months. A total of 2 patients remained alive at the last follow-up (32 and 300 months, respectively). The present study demonstrated that brain metastases may occur in $\leq 6 \%$ of patients with NMTC and distant metastases. Brain metastases rarely present at diagnosis of NMTC and are associated with metastases in
\end{abstract}

Correspondence to: Dr Ilana Slutzky-Shraga, Endocrine Institute, Rabin Medical Center, Beilinson Hospital, 39 Jabotinski, Petach Tikva 4941492, Israel

E-mail: shragas2@clalit.org.il

Key words: thyroid cancer, non-medullary, distant metastases, brain metastases, disease outcome other distant sites. Systematic screening for brain metastases requires consideration in all patients with NMTC and distant metastases. Some patients show an indolent evolution with overall survival of $>2$ years, supporting an aggressive treatment approach.

\section{Introduction}

Non-medullary thyroid carcinoma (NMTC) originating from thyroid epithelial cells accounts for $>90 \%$ of all thyroid malignancies (1). Patients with NMTC usually demonstrate a good prognosis, with a 10 -year survival rate of $80-95 \%$ (2). However, 4-23\% of patients will present with distant metastases (DM) at the time of diagnosis or over the course of their disease (3-8). Our previous study reported a $6.8 \%$ rate of DM for all patients with NMTC who were treated and followed-up at our institution (9). The presence of DM is associated with a poor prognosis, constituting the most frequent cause of thyroid cancer-associated mortality (8-10). The most common sites of DTC metastases are the lungs and the bones, followed by the brain and the liver (11). While the 10-year survival rate in the whole population of NMTC patients with DM may reach $~ 50 \%$ (12), intracranial DM are associated with a median survival time of $<1$ year $(13,14)$. Data on the optimal management of brain metastases in this setting and the factors affecting outcome remain limited. In potentially curable patients with isolated brain metastases, surgical excision is indicated, while the administration of 131-iodine therapy for radioiodine-responsive disease and external-beam radiation therapy (EBRT) may partially control disease progression in selected patients. Data regarding the role of systemic therapy with kinase inhibitors are limited (15).

Furthermore, rates of brain metastases occurrence may be underestimated. While patients may present with headaches or various neurological symptoms, intracranial metastasis may be asymptomatic. According to a previous study, asymptomatic brain metastases were diagnosed incidentally during systemic brain imaging prior to prescription of tyrosine kinase inhibitors in $15.4 \%$ of patients with NMTC and distant metastases (13). De Figueiredo et al (13) suggested, in agreement with others (16-19), that surgical intervention, EBRT and 
stereotactic radiosurgery (SRS) may prolong survival in this group of patients.

The aim of the present study was to evaluate a series of patients with NMTC-associated brain metastases who were treated and subsequently followed-up at a single tertiary medical center.

\section{Patients and methods}

The electronic database of the Rabin Medical Center Thyroid Cancer Registry and the Head and Neck Tumor Unit, Beilinson Hospital (Petach Tikva, Israel) was searched for all patients diagnosed and treated for NMTC with distant metastases between 1970 and 2014. A total of 10 patients with brain metastases, confirmed by computerized tomography or magnetic resonance imaging, were identified and formed the study group. Patients with anaplastic cancer were excluded. The medical records of the eligible patients were reviewed for data on clinical characteristics, histological type of primary tumor, sites of distant metastases, diagnosis and treatment of brain metastases, time intervals for diagnosis and treatment of the brain metastasis, disease outcome and tumor-node-metastasis staging of the thyroid cancer, according to the American Joint Committee on Cancer (seventh edition) (20). The study protocol was approved by the Institutional Ethics Board of Rabin Medical Center, Beilinson Hospital.

Statistical analysis. Categorical variables are presented as numbers and percentage, and continuous variables as means and standard deviations or medians with ranges. Categorical variables were compared using $\chi^{2}$ test, and continuous variables using unpaired Student's t-test or Mann Whitney U-test, as appropriate. The product-limit estimate method (Kaplan-Meier) was used to estimate survival in the cohort of patients with distant metastases, and the log-rank test was used to compare survival curves. $\mathrm{P}<0.05$ was considered to indicate a statistically significant difference. All analyses were conducted using SAS Software, version 9.4 (SAS Institute, Inc., Cary, NC, USA).

\section{Results}

Baseline patient characteristics and clinicopathological findings. Clinicopathological characteristics of patients are presented in Table I. Of the 172 patients diagnosed and treated for NMTC with distant metastases during the study period, $10(5.8 \%)$ possessed brain metastases. This included 6 female and 4 male patients with a median age of 53.5 years (range, 18-81 years). The median follow-up time from primary diagnosis was 84 months (7 years; range, 1-25 years). Histological analysis of the primary tumor revealed papillary thyroid carcinoma (PTC)-classical variant in 3 patients, and PTC-follicular variant in 3 patients. The remaining 4 patients exhibited an aggressive variant, including poorly differentiated and follicular in 2 , tall cell in 1, and insular in 1 patient. Tumor stage was determined to be pT4 in 5 patients, pT3 in 2 patients, pT 2 in 2 patients and unknown in the remaining patient (20). Extra-thyroidal extension was documented in $7 / 9$ patients $(78 \%)$. A total of 6 patients $(60 \%)$ demonstrated nodal involvement, and $5(50 \%)$ possessed distant metastases at diagnosis.

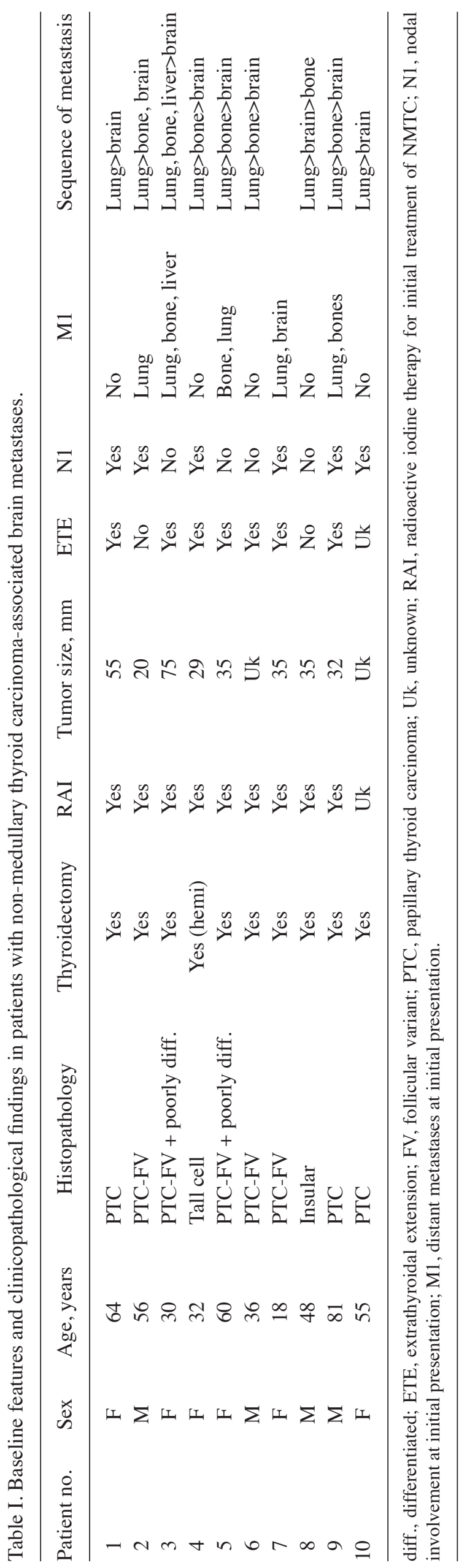



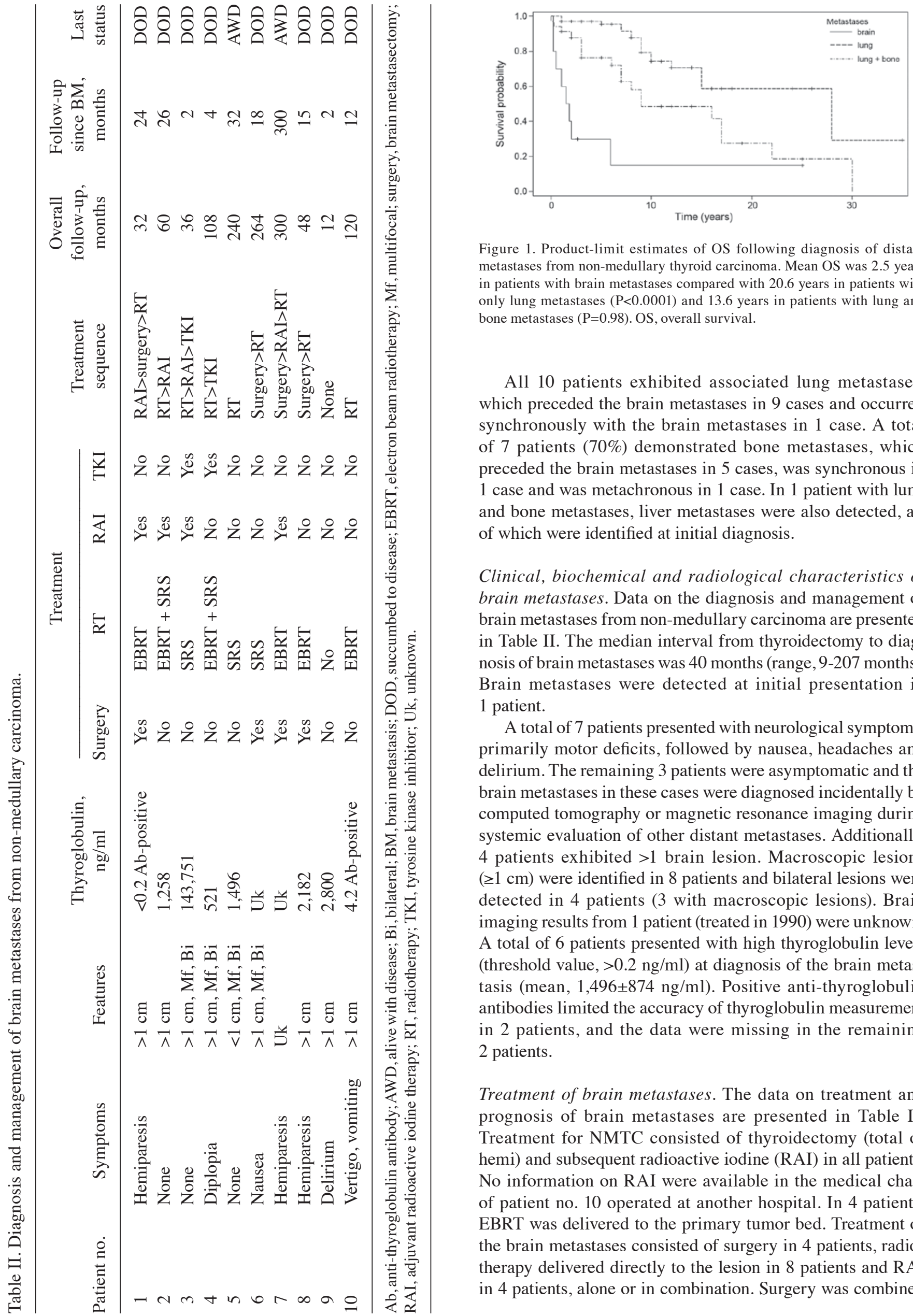

Figure 1. Product-limit estimates of OS following diagnosis of distant metastases from non-medullary thyroid carcinoma. Mean OS was 2.5 years in patients with brain metastases compared with 20.6 years in patients with only lung metastases $(\mathrm{P}<0.0001)$ and 13.6 years in patients with lung and bone metastases $(\mathrm{P}=0.98)$. OS, overall survival.

All 10 patients exhibited associated lung metastases, which preceded the brain metastases in 9 cases and occurred synchronously with the brain metastases in 1 case. A total of 7 patients $(70 \%)$ demonstrated bone metastases, which preceded the brain metastases in 5 cases, was synchronous in 1 case and was metachronous in 1 case. In 1 patient with lung and bone metastases, liver metastases were also detected, all of which were identified at initial diagnosis.

Clinical, biochemical and radiological characteristics of brain metastases. Data on the diagnosis and management of brain metastases from non-medullary carcinoma are presented in Table II. The median interval from thyroidectomy to diagnosis of brain metastases was 40 months (range, 9-207 months). Brain metastases were detected at initial presentation in 1 patient.

A total of 7 patients presented with neurological symptoms, primarily motor deficits, followed by nausea, headaches and delirium. The remaining 3 patients were asymptomatic and the brain metastases in these cases were diagnosed incidentally by computed tomography or magnetic resonance imaging during systemic evaluation of other distant metastases. Additionally, 4 patients exhibited $>1$ brain lesion. Macroscopic lesions $(\geq 1 \mathrm{~cm})$ were identified in 8 patients and bilateral lesions were detected in 4 patients ( 3 with macroscopic lesions). Brain imaging results from 1 patient (treated in 1990) were unknown. A total of 6 patients presented with high thyroglobulin levels (threshold value, $>0.2 \mathrm{ng} / \mathrm{ml}$ ) at diagnosis of the brain metastasis (mean, $1,496 \pm 874 \mathrm{ng} / \mathrm{ml}$ ). Positive anti-thyroglobulin antibodies limited the accuracy of thyroglobulin measurement in 2 patients, and the data were missing in the remaining 2 patients.

Treatment of brain metastases. The data on treatment and prognosis of brain metastases are presented in Table II. Treatment for NMTC consisted of thyroidectomy (total or hemi) and subsequent radioactive iodine (RAI) in all patients. No information on RAI were available in the medical chart of patient no. 10 operated at another hospital. In 4 patients, EBRT was delivered to the primary tumor bed. Treatment of the brain metastases consisted of surgery in 4 patients, radiotherapy delivered directly to the lesion in 8 patients and RAI in 4 patients, alone or in combination. Surgery was combined 
with EBRT in 1 patient, with SRS in 1, and with EBRT and RAI in 2. One patient each underwent only EBRT or SRS, and 1, ERBT + SRS and RAI. Patient no. 3 received tyrosine kinase inhibitors in combination with SRS and RAI, and patient no. 4 in combination with radiotherapy. In patient no. 9 , the brain metastases were not treated due to a poor performance status. The thyroid was confirmed by histology to be the origin of the brain metastases in 4 patients following surgical resection.

Disease outcomes. Median overall survival time following diagnosis of brain metastasis was 15 months (range, 2-59 months). A total of 6 patients survived for $>12$ months, of whom 2 remained alive at their last follow-up (duration of follow-up from brain metastases diagnosis, 32 and 300 months, respectively). Mortality in the remaining patients was due to progression of the brain lesions in 5 patients and worsening of pulmonary metastases in 3 patients.

Median survival time was markedly increased in patients who underwent surgical resection and/or external radiotherapy (28 months) compared with patients who did not (4 months). The small sample size of the study precluded an analysis of survival by type of treatment. Mean survival time in the patients with brain metastases ( 2.5 years) was significantly decreased compared with the other patients with NMTC with distant metastases only in the lungs (20.6 years; $\mathrm{P}<0.0001)$ (Fig. 1).

Patient no. 7 (Tables I and II) was the only patient in whom brain metastasis was diagnosed prior to diagnosis of the primary tumor. Following total thyroidectomy and radioiodine treatment, the patient underwent five neurosurgeries followed by EBRT. Despite severe disability, the patient received a bachelor's degree in social work and, at the age of 41 years, successfully gave birth by caesarean section. She remains alive, after a follow-up of 25 years.

\section{Discussion}

The present study demonstrated that the brain is a relatively frequent site of secondary metastases in patients with NMTC and distant metastases, reaching rates of $\sim 6 \%$. This finding is in agreement with previous studies comprising large cohorts of $>600$ patients with NMTC (21-25), including our previous study (9). In the present study, all the patients exhibited lung metastases and the majority also possessed bone metastases. Hematogenic spread of tumor cells may explain the association observed between intracranial involvement and lung metastases. Notably, a high rate of extrathyroidal extension (78\%) was observed in this cohort of patients. As such, clinicians should consider this risk and investigate the possibility of intracranial involvement as early as possible in patients with NMTC and distant metastases in order to minimize clinical deterioration. The considerable proportion $(30 \%)$ of asymptomatic brain lesions in the present study justifies the use of brain imaging in all patients with disseminated disease.

Serum thyroglobulin levels were high $(1,496 \pm 874 \mathrm{ng} / \mathrm{ml})$ in all patients with brain metastases who were negative for anti-thyroglobulin antibodies, confirming the differentiated nature of the thyroid cancer. Practically, however, a high thyroglobulin level is a problematic indicator to suspect brain metastases given that all the patients in the present study had distant metastases in other sites. For the same reason, high serum thyroglobulin levels cannot serve as an indicator of response to therapy.

The median survival time of 15 months in the present cohort is longer compared with those reported in other studies, namely 7.1 months (13), 9.4 months (25) and 12.4 months (24). This difference in survival time may be attributable to the relatively young age of the patients in the present study (median, 53.5 years) compared with those in previous studies (with median ages of 59 and 63 years) $(13,17,24,26)$, the exclusion of patients with anaplastic-type cancer from the present study, and the aggressive treatment approach employed in the present study. For example, patient no. 7 underwent five neurosurgeries in addition to EBRT and RAI. The use of stereotactic radiotherapy in patients with a limited number of brain foci has been increasing, because it allows for the delivery of a large dose to the target mass while minimizing damage to normal brain tissue (17). Treatment with SRS alone or in addition to whole-body radiation therapy has been demonstrated to achieve a local control rate of $96 \%$ without any procedure-associated complications (18). In the present study cohort, the patients who were treated for brain metastases with surgery and/or radiation achieved a longer survival time compared with untreated patients (patient nos. 4 and 9), similar to previous studies $(13,17,18)$. In a recent study by Choi et al (26), the median overall survival time following diagnosis of brain metastases was 16.7 months in treated patients compared with 2.6 months in untreated patients.

The results of the present study should be interpreted with caution due to the retrospective design of the study and the inclusion of only a small sample of patients from a single institution. However, due to the rarity of NMTC with brain metastases and the lack of data from prospective studies, management decisions currently rely on case reports and retrospective studies (23). The present study is of interest due to the improved outcome achieved in the aggressively treated patients, the high association observed between brain metastases and lung metastases in NMTC and the high incidence of incidentally diagnosed brain metastases.

In conclusion, the present study demonstrates that a higher-than-expected proportion of patients with lung and bone metastases from NMTC also exhibit brain metastases. Therefore, systemic screening should be considered for all patients with disseminated NMTC. Surgical resection of all operable metastatic lesions with multidisciplinary adjuvant treatment is critical to improve patient survival.

\section{References}

1. Chen AY, Jemal A and Ward EM: Increasing incidence of differentiated thyroid cancer in the united states, 1988-2005. Cancer 115: 3801-3807, 2009.

2. Schlumberger MJ: Papillary and follicular thyroid carcinoma. N Engl J Med 338: 297-306, 1998.

3. Massin JP, Savoie JC, Garnier H, Guiraudon G, Leger FA and Bacourt F: Pulmonary metastases in differentiated thyroid carcinoma. Study of 58 cases with implications for the primary tumor treatment. Cancer 53: 982-992, 1984.

4. Ruegemer JJ, Hay ID, Bergstralh EJ, Ryan JJ, Offord KP and Gorman CA: Distant metastases in differentiated thyroid carcinoma: A multivariate analysis of prognostic variables. J Clin Endocrinol Metab 67: 501-508, 1988. 
5. Casara D, Rubello D, Saladini G, Gallo V, Masarotto G and Busnardo B: Distant metastases in differentiated thyroid cancer: Long-term results of radioiodine treatment and statistical analysis of prognostic factors in 214 patients. Tumori 77: 432-436, 1991.

6. Samaan NA, Schultz PN, Haynie TP and Ordonez NG: Pulmonary metastasis of differentiated thyroid carcinoma: Treatment results in 101 patients. J Clin Endocrinol Metab 60: 376-380, 1985.

7. Jonklaas J, Sarlis NJ, Litofsky D, Ain KB, Bigos ST, Brierley JD, Cooper DS, Haugen BR, Ladenson PW, Magner J, et al: Outcomes of patients with differentiated thyroid carcinoma following initial therapy. Thyroid 16: 1229-1242, 2006.

8. Lang BH, Wong KP, Cheung CY, Wan KY and Lo CY: Evaluating the prognostic factors associated with cancer specific survival of differentiated thyroid carcinoma presenting with distant metastasis. Ann Surg Oncol 20: 1329-1335, 2013.

9. Hirsch D, Levy S, Tsvetov G, Gorstein A, Slutzky-Shraga I, Akirov A, Robenshtok E, Shimon I and Benbassat CA: Long term outcomes and prognostic factors in patients with differentiated thyroid cancer and distant metastases. Endocr Pract: 13 Jul, 2017 (Epub ahead of print).

10. Dinneen SF, Valimaki MJ, Bergstralh EJ, Goellner JR, Gorman CA and Hay ID: Distant metastases in papillary thyroid carcinoma: 100 cases observed at one institution during 5 decades. J Clin Endocrinol Metab 80: 2041-2045, 1995.

11. Hoie J, Stenwig AE, Kullmann G and Lindegaard M: Distant metastases in papillary thyroid cancer. A review of 91 patients. Cancer 61: 1-6, 1988.

12. Clark JR, Lai P, Hall F, Borglund A, Eski S and Freeman JL: Variables predicting distant metastases in thyroid cancer. Laryngoscope 115: 661-667, 2005.

13. Henriques de Figueiredo B, Godbert Y, Soubeyran I, Carrat X, Lagarde P, Cazeau AL, Italiano A, Sargos P, Kantor G, Loiseau H and Bonichon F: Brain metastases from thyroid carcinoma: A retrospective study of 21 patients. Thyroid 24: 270-277, 2014.

14. Samuel AM and Shah DH: Brain metastases in well-differentiated carcinoma of the thyroid. Tumori 83: 608-610, 1997

15. Haugen BR, Alexander EK, Bible KC, Doherty GM, Mandel SJ, Nikiforov YE, Pacini F, Randolph GW, Sawka AM, Schlumberger M, et al: 2015 American thyroid association management guidelines for adult patients with thyroid nodules and differentiated thyroid cancer: The american thyroid association guidelines task fork on thyroid nodules and differentiated thyroid cancer. Thyroid 26: 1-133, 2016

16. Salvati M, Frati A, Rocchi G, Masciangelo R, Antonaci A, Gagliardi FM and Defini R: Single brain metastasis from thyroid cancer: Report of twelve cases and review of the literature. J Neurooncol 51: 33-40, 2001.
17. Bernard DM, Sperduto PW, Souhami L, Jensen AW and Roberge D: Stereotactic radiosurgery in the management of brain metastases from primary thyroid cancers. J Neurooncol 98: 249-252, 2010

18. Kim IY, Kondziolka D, Niranjan A, Flickinger JC and Lunsford LD: Gamma knife radiosurgery for metastatic brain tumors from primary thyroid cancers. J Neurooncol 93: 355-359, 2009.

19. McWilliams RR, Giannini C, Hay ID, Atkinson JL, Stafford SI and Buckner JC: Management of brain metastases from thyroid carcinoma: A study of 16 pathologically confirmed cases over 25 years. Cancer 98: 356-362, 2003.

20. Edge $S$ and Compton C: The american joint committee on cancer: The seventh edition of the AJCC cancer staging manual and the future of TNM. Ann Surg Oncol 17: 1471-1474, 2010.

21. Kim KM, Park JB, Bae KS and Kang SJ: Analysis of prognostic factors in patients with multiple recurrences of papillary thyroid carcinoma. Surg Oncol 21: 185-190, 2012.

22. Nixon IJ, Whitcher MM, Palmer FL, Tuttle MR, Shaha AR, Shan JP, Patel SG and Ganly I: The impact of distant metastases at presentation on prognosis in patients with differentiated carcinoma of the thyroid gland. Thyroid 22: 884-889, 2012.

23. Hay ID: Brain metastases from papillary thyroid carcinoma. Arch Intern Med 147: 607-611, 1987.

24. Chiu AC, Delpassand ES and Sherman SI: Prognosis and treatment of brain metastases in thyroid carcinoma. J Clin Endocrinol Metab 82: 3637-3642, 1997

25. Misaki T, Iwata M, Kasagi K and Konishi J: Brain metastases from differentiated thyroid cancer in patients treated with radioiodine for bone and lung lesions. Ann Nucl Med 14: 111-114, 2000.

26. Choi J, Kim JW, Keum YS and Lee IJ: The largest known survival analysis of patients with brain metastasis from thyroid cancer based on prognostic groups. PLoS One 11: e0154739, 2016.

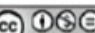

reative Commons Attribution-NonCommercial-NoDerivatives 4.0 International (CC BY-NC-ND 4.0) License. 